Review/Meta-Analyses

\title{
A systematic review on sleep alterations anticipating the onset of bipolar disorder
}

\author{
C. Pancheri ${ }^{\mathrm{a}, \mathrm{b}}$, N. Verdolini ${ }^{\mathrm{b}, \mathrm{c}}$, I. Pacchiarotti ${ }^{\mathrm{b}}$, L. Samalin ${ }^{\mathrm{d}}$, R. Delle Chiaie ${ }^{\mathrm{a}}$, M. Biondi ${ }^{\mathrm{a}}$, \\ A.F. Carvalho ${ }^{\text {e,f }}$, M. Valdes ${ }^{g}$, P. Ritter ${ }^{\text {h }}$, E. Vieta ${ }^{\text {b,* }}$, A. Murru ${ }^{\text {b }}$ \\ a Department of Neurology and Psychiatry, Policlinico Umberto I, La Sapienza University of Rome, Rome, Italy \\ ${ }^{\mathrm{b}}$ Bipolar and Depressive Disorders Unit, Institute of Neuroscience, Hospital Clinic, University of Barcelona, IDIBAPS, CIBERSAM, 170 Villarroel st, 12-0, 08036, \\ Barcelona, Catalonia, Spain \\ ${ }^{\mathrm{c}}$ Division of Psychiatry, Clinical Psychology and Rehabilitation, Department of Medicine, Santa Maria della Misericordia Hospital, University of Perugia, Italy \\ d CHU Clermont-Ferrand, Department of Psychiatry, EA7280, University of Auvergne, Clermont-Ferrand, France \\ e Department of Psychiatry, Faculty of Medicine, University of Toronto, Toronto, ON, Canada \\ ${ }^{\mathrm{f}}$ Centre for Addiction \& Mental Health (CAMH), Toronto, ON, M6J 1H4, Canada \\ ${ }^{g}$ Department of Psychiatry and Psychology, Institute of Neuroscience, Hospital Clínic, University of Barcelona, Villarroel 170, Barcelona, Spain \\ ${ }^{\mathrm{h}}$ Department of Psychiatry and Psychotherapy, Carl Gustav Carus University Hospital, Technische Universität Dresden, Dresden, Germany
}

\section{A R T I C L E IN F O}

\section{Article history:}

Received 6 September 2018

Received in revised form 23 January 2019

Accepted 8 February 2019

Available online 25 February 2019

\section{Keywords:}

Sleep symptoms

Sleep prodromes

Bipolar disorder

Review

\begin{abstract}
A B S T R A C T
Background: Sleep alterations are frequent occurrence in Bipolar Disorder (BD), both in acute and interepisodic phases. Sleep alterations have been also described both long before BD onset, as aspecific risk syndromes, or as immediate prodromes of $\mathrm{BD}$ onset. The aim of the present study is to systematically review the relationship between sleep alterations anticipating for the full-blown onset of BD, both in general and according to specific polarities of onset.

Methods: A systematic literature research according to PRISMA statement and considering: 1. prospective studies about BD patients' offspring with sleep alterations who later developed BD. 2. prospective studies assessing patients with sleep disorders who later developed BD. 3. retrospective studies on BD patients where sleep alterations before BD onset of the disease were reported.

Results: A total of 16 studies were included in this review. Sleep disturbances may frequently appear 1 year before the onset of $\mathrm{BD}$ or more, often during childhood or adolescence. A decreased need for sleep may precede the onset of the illness, specially a manic episode, while insomnia appears to anticipate either a manic or a depressive episode. Hypersomnia seems to precede bipolar depressive episodes. Conclusions: Sleep alterations frequently appear long before the onset of BD, and appear to be related specifically to the polarity of the index episode. The detection and treatment of sleep alterations in special high risk populations may help achieving an earlier detection of the illness.
\end{abstract}

(ㄷ) 2019 Elsevier Masson SAS. All rights reserved.

\section{Introduction}

Chronic and recurrent conditions such as bipolar disorder (BD) show improved short- and long-term outcome when early detection and treatment is provided [1-3]. The identification of risk factors or prodromal symptoms may help defining at-risk stages before full-blown syndromic presentations with major clinical and treatment implications and, ideally allowing for early stage-specific, rather than disorder-specific, treatments [4,5].

\footnotetext{
* Corresponding author at: Bipolar Disorder Unit, IDIBAPS CIBERSAM, Hospital Clínic de Barcelona, c/Villaroel 170, escalera 12 planta 0, 08036 Barcelona, Spain. E-mail address: evieta@clinic.ub.es (E. Vieta).
}

Sleep abnormalities have been proposed as susceptibility markers in individuals at high risk of developing BD [6,7]. They are included as diagnostic criteria and almost invariably present during acute episodes [8]. Sleep abnormalities are frequently present as immediate or quasi-immediate prodromes of BD acute episodes [9-13].

Circadian abnormalities and sleep symptoms represent a cardinal feature during acute manic and depressive episodes, potentially serving as trait biomarkers of BD [8]. BD patients suffer from enduring biological rhythm abnormalities, even during remission phases $[10,14]$. Correlations between several sleep and circadian phenotypes, and the development of BD were demonstrated, providing clues to new approaches for both preventing and treating $\mathrm{BD}[10,15]$. 
Sleep alterations, might also precede the onset of BD by presenting as risk syndromes, detectable before the full-blown mood disorder, or as prodromes immediately preceding the first acute mood episode. Both as risk factors or immediate prodromes, detectable sleep alterations before first- or following affective episodes sahre implications for clinical research and practice [16], but also for early diagnosing [17]. Moreover, the possibility of objective measures of sleep alterations outlines their potential role as biomarkers, helping to redefine BD beyond a classical clinical definition [18,19].

In recent years, a systematic review focused on a possible link between the onset of sleep problems and the subsequent development of BD [20], but its results were somewhat hampered by a relative scarcity of studies reporting prospective results.

Considering the growing evidence on this topic in later years, our aim was to perform and updated systematic review on the evidence on the possible role of sleep alterations anticipating the onset of BD. Secondarily, the aim of this review is to investigate the possible relationship between specific sleep alterations according to the polarity of the BD acute affective onset.

\section{Methods}

The present review has been conducted according to the PRISMA (Preferred Reporting Items for Systematic Reviews and Meta-Analyses) statement [21]. Studies focused on sleep alterations preceding BD relapses/recurrences and studies including individuals at high risk for BD not meeting a standardized diagnosis were not considered in the present review.

\subsection{Literature Review}

A systematic search was performed using MEDLINE/PubMed/ Index Medicus and the Cochrane Library, considering a time period ending on January $1^{\text {st }}, 2018 \mathrm{~A}$ cross-check between references obtained was done. A further search in the site www.clinicaltrials. gov controlled for literature results and screened for eventually started, ongoing, or concluded but unpublished studies.

Studies included were: 1. Prospective studies on offspring of BD patients, later diagnosed with $\mathrm{BD} ; 2$. Prospective studies on patients with sleep problems developing BD; Retrospective studies on sleep problems in BD patients.

The full search strategy for the 3 types of studies included is presented in the S2.1 file

\subsection{Eligibility criteria}

Studies should report details on design, sample description, inclusion/exclusion criteria, defined aims, clear methodological procedures, clear outcome definitions or information about the diagnostic tools used for the assessments, and considering BD individuals diagnosed with DSM-IV-TR/DSM-IV/DSM-III [22-24] or ICD-10/ICD-9/ICD-8 [25-27].

\subsubsection{Inclusion criteria}

Original peer-reviewed articles published in English language.

2.2.2. Specific inclusion criteria for the 3 types of studies included in the present review

Prospective studies on offspring of BD patients:

1 Cohort studies on high-risk individuals (i.e. offspring or unaffected first-degree relatives of individuals with $\mathrm{BD}$, and had assessed sleep alterations with/without circadian alterations at baseline, and conversion to full-blown BD at follow-up.
Prospective studies on patients with sleep problems developing BD:

2 Cohort studies on individuals with sleep alterations with/ without circadian alterations and psychopathology assessment at baseline, and the development of full-blown BD at follow-up.

Retrospective studies on BD patients:

3 Cohort studies on individuals with a diagnosis of BD or a first episode of psychosis later diagnosed with $\mathrm{BD}$, studying sleep alterations with/without circadian alterations at baseline (working definitions for this condition vary, but studies in which a specific definition was included were considered for inclusion). Study sample size of at least 30 individuales. These studies should then report specific data on conversion to fullblown BD at follow-up.

Sleep alterations could be assessed via either questionnaire or through actigraphy or polysomnography or other specific sleeprelated exams.

Results on sleep alterations in BD patients with depressive or psychotic onset were considered for inclusion when a further, subsequent conversion to a BD diagnosis was established with standard assessments.

\subsubsection{Exclusion criteria}

Exclusion criteria were: 1 . animal studies; 2 . Studies including general or psychiatric populations not specifying the number of BD individuals; 3. Studies not specifying a standardized BD diagnosis (criteria DSM or ICD) at baseline and/or follow-up; 4. Studies with no specific sleep assessment; 5 . Reviews or meta-analyses or studies with $\mathrm{N}<10$. 6. Studies focused on sleep alterations preceding BD relapses/recurrences. 7. Studies including individuals at high risk for BD who did not meet a standardized diagnosis.

\subsection{Data collection process and items}

Data were classified into three groups: 1. data of interest, 2. duplicated data, and 3. data of no interest, according to the previously described criteria. Studies concerning molecular and genetic aspects, as well as those assessing or comparing efficacy of medications, were not included.

After first exclusion, full studies were retrieved and examined. References were also reviewed to identify further possible studies of interest.

\subsubsection{Data extraction}

The titles and/or abstracts of studies were screened independently ( $\mathrm{CP}$ and $\mathrm{NV}$ ) to identify studies that potentially met the inclusion criteria outlined above. After elimination of duplicated sources, the full texts of these potentially eligible studies were retrieved and independently assessed for eligibility by $\mathrm{CP}$ and NV. Any disagreement over the eligibility of particular studies was resolved through consensus or, when agreement was not achieved, through discussion with AM. A standardized, excel form was used to extract data from the included studies for the assessment of study quality and for evidence synthesis. Where possible, time between first sleep symptoms and onset of BD was extracted and presented along with other results.

\subsubsection{Methodological quality assessment}

Two independent authors ( $\mathrm{CP}$ and NV) evaluated the risk of bias for each domain described in the Newcastle-Ottawa Scale (NOS) which was developed to assess the quality of non-randomized case-control and cohort studies with its design, content and ease of 
use directed to the task of incorporating the quality assessments in the interpretation of meta-analytic results [28]. For cross-sectional studies, a previously adapted version of the NOS was used [29]. Discrepancies between the two raters were solved by consensus among three researchers (CP, NV, AM).

\section{Results}

\subsection{Systematic search results}

The whole search returned 17,886 papers. After duplicate removal, 14,572 papers were excluded on the basis of title or abstract because of no direct interest. The remaining 285 studies were examined and assessed for inclusion, of them 268 were excluded for different reasons (Shared causes: Studies without specific assessment points on sleep: 50; Studies without psychopathological items at baseline or specified diagnosis of BD: 70; Studies do not grant access data: 2; Studies with sample already included: 5. Specific causes (prospective and retrospective studies): No prospective studies, respectively: 99; No retrospective studies: 42). Finally, 17 studies were included in the present review (Fig. 1).

\subsection{Contents results}

\subsubsection{BD offspring prospective studies}

A total of 4 studies were included in this part of the review (Table 1).

The CARE (Children and Adolescent Research Evaluation) study is a prospective follow-up cohort on healthy Amish started in 1994, and aimed at detecting possible prodromal features for BD [30] After 16 years of follow-up, 9 children met full criteria for BD type I, 8 originally from the BD parent sample, 1 from the control sample.
At the last follow-up 50\% of children later diagnosed with BD presented decreased sleep as an antecedent.

A Canadian prospective study began in 1998 with the aim to describe the possible onset of psychiatric disorders in siblings of BD parents [31]. BD diagnosis was met by 31 subjects, all from the high-risk offspring. The high-risk offspring had a higher lifetime risk of sleep disorders $(\mathrm{HR}=28.21, \mathrm{P}=0.02)$, compared with control offspring. Anxiety/sleep disorders started about 6 years before the onset of first major mood episode $(p=0.008)$.

In the Pittsburgh Bipolar Offspring study (BIOS) [32], BD parents and healthy comparison parents were recruited. All offspring of $6-$ 18 years of age were included. Offspring of BD parents ("at-risk"). A sample of 612 (335 BD and 277 non-BD) offspring were assessed until age 18. At the final $8^{\text {th }}$-year endpoint, 25/227 high-risk offspring developed BD. Three latent sleep groups were characterized (good sleepers, poor sleepers and variable sleepers) based on the likelihood of sleep. After controlling for confounding factors, the poor sleep group had a 4-fold odds of developing BD than those in the good sleep group $(\mathrm{OR}=4.25)$, though results remained marginally non-significant. High "weekend-to-weekdays variability" sleep patterns (variable group) had low likelihood of developing BD. Sleep symptoms presented about 3 years before BD onset.

The Dutch Bipolar Offspring Study [33] aimed to identify early signs of mood disorders, specifically BD in adolescent BD offspring. At baseline, $27 \%$ of the offsprings had a history of any mood disorder and 73\% had no mood diagnosis ("No MD"). After 12 years, $10 / 29$ (34\%) from the 'Any MD' group converted to BD with an (hypo)manic onset. In this group, decreased need of sleep (50\% vs $5 \%, \mathrm{p}=0.011)$ and middle insomnia ( $40 \%$ vs $5 \%, \mathrm{p}=0.036)$ were significantly associated with BD conversion. The median time to (hypo)mania onset after the baseline assessment was 2.7 years.

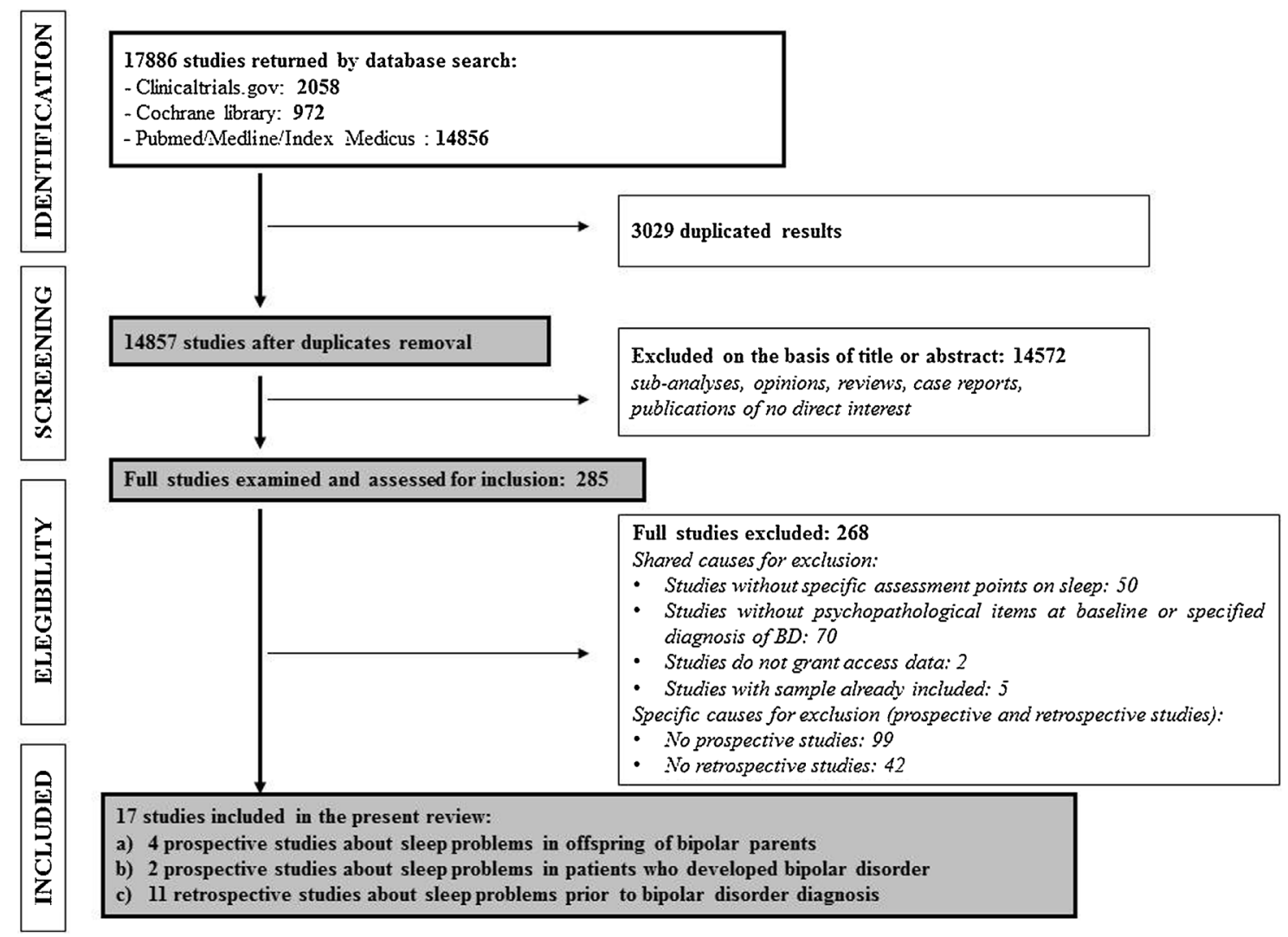

Fig. 1. PRISMA flowchart of studies selection for the systematic review. 
Table 1

Prospective studies of offspring with parents with Bipolar Disorder.

\begin{tabular}{|c|c|c|c|c|c|c|c|c|c|c|c|c|}
\hline \multirow[b]{2}{*}{ Study } & \multicolumn{2}{|l|}{ Design details } & \multicolumn{3}{|c|}{ Assessment } & \multirow[b]{2}{*}{$\begin{array}{l}\text { Follow- } \\
\text { up } \\
\text { (years) }\end{array}$} & \multicolumn{3}{|l|}{$\begin{array}{l}\text { Age } \\
\text { (years) }\end{array}$} & \multirow[b]{2}{*}{$\begin{array}{l}\text { Type } \\
1^{\text {st }} \\
\text { episode }\end{array}$} & \multicolumn{2}{|l|}{ Sleep prodromes } \\
\hline & Cohort & Sample & $\begin{array}{l}\text { Parents } \\
\text { diagnosis } \\
\text { / scales }\end{array}$ & $\begin{array}{l}\text { Offspring } \\
\text { diagnosis / } \\
\text { scales }\end{array}$ & Sleep & & Baseline & $\begin{array}{l}\text { Sleep } \\
\text { problems }\end{array}$ & $\begin{array}{l}1^{\text {st }} \\
\text { episode }\end{array}$ & & Type & $\begin{array}{l}\text { Preceding } \\
\text { BD }\end{array}$ \\
\hline $\begin{array}{c}\text { Egeland } \\
2012\end{array}$ & CARE /Amish & $\begin{array}{l}\text { HR: } 15 \\
\text { families } \\
\text { /115 } \\
\text { children } \\
\text { Controls: } \\
12 \text { families } \\
\text { /106 } \\
\text { children }\end{array}$ & $\begin{array}{l}\text { BD-I / } \\
\text { CARE } \\
\text { interview }\end{array}$ & $\begin{array}{l}\text { BD-I } \\
\text { / CARE } \\
\text { interview }\end{array}$ & $\begin{array}{l}\text { CARE } \\
\text { interview }\end{array}$ & 16 & $<14$ & NS & 15.1 & NS & $\begin{array}{l}-\downarrow \text { sleep } \\
\text {-Difficulty falling } \\
\text { asleep } \\
\text {-Early morning } \\
\text { awakening }\end{array}$ & NS \\
\hline $\begin{array}{r}\text { Duffy } \\
2014\end{array}$ & $\begin{array}{l}\text { Outpatient } \\
\text { clinic / } \\
\text { Canada }\end{array}$ & $\begin{array}{l}\text { HR: } 229 \\
\text { Control: } 86\end{array}$ & $\begin{array}{l}\text { BD-I BD-II } \\
\text { / SADS-L }\end{array}$ & $\begin{array}{l}\text { BD-I, BD-II, } \\
\text { SZA } \\
\text { / KSADS-PL, } \\
\text { SADS-L }\end{array}$ & $\begin{array}{l}\text { Items } \\
\text { from } \\
\text { KSADS- } \\
\text { PL, SADS- } \\
\text { L }\end{array}$ & 15 & $16.3 \pm 5.3$ & $\begin{array}{l}\text { (median) } \\
9.8\end{array}$ & $\begin{array}{l}>20.8 \mathrm{D} \\
\text { onset } \\
>15.5 \mathrm{M} / \\
\text { (h)M } \\
\text { onset }\end{array}$ & $\begin{array}{l}83.9 \% \mathrm{D} \\
16.1 \% \\
\mathrm{M} /(\mathrm{h}) \\
\mathrm{M}\end{array}$ & -Sleep disorders & $\sim 6 y^{*}$ \\
\hline $\begin{array}{l}\text { Levenson } \\
2017\end{array}$ & BIOS / USA & $\begin{array}{l}\text { HR: } 335 \\
\frac{\text { Control: }}{277}\end{array}$ & $\begin{array}{l}\text { BD-I BD-II } \\
\text { / SCID }\end{array}$ & $\begin{array}{l}\text { BD } \\
\text { Spectrum / } \\
\text { SCID, } \\
\text { KSADS-PL,K- } \\
\text { SADS }\end{array}$ & SSHS & 8 & $12.8 \pm 2.2$ & $14.3 \pm 1.8$ & $17.2 \pm 3$ & NS & $\begin{array}{l}\text { - Poor sleepers: } \\
\text { moderate }(0.40) \text { to } \\
\text { high }(>0.70) \\
\text { incidence of sleep } \\
\text { deficiencies in } \\
\text { nearly all of the sleep } \\
\text { domains }\end{array}$ & $\sim 3 y^{*}$ \\
\hline $\begin{array}{c}\text { Mesman } \\
2017\end{array}$ & $\begin{array}{l}\text { Dutch } \\
\text { Bipolar } \\
\text { Offspring } \\
\text { Study / } \\
\text { Germany }\end{array}$ & HR: 107 & $\begin{array}{l}\text { BD-I BD- } \\
\text { II/ SCID }\end{array}$ & $\begin{array}{l}\text { BD } \\
\text { Spectrum / } \\
\text { SCID, } \\
\text { KSADS-PL }\end{array}$ & $\begin{array}{l}\text { Items } \\
\text { from K- } \\
\text { SADS-PL }\end{array}$ & 12 & $\begin{array}{l}16(12- \\
21)\end{array}$ & $\begin{array}{l}16(12- \\
21)\end{array}$ & $\begin{array}{l}18.7(h) \\
\text { M onset } \\
20.9 \mathrm{D} \\
\text { onset }\end{array}$ & $\begin{array}{l}34 \%(h) \\
M \\
2.6 \% \mathrm{D}\end{array}$ & $\begin{array}{l}\text { - Decreased need of } \\
\text { sleep } \\
\text { - Middle insomnia }\end{array}$ & $2.7 \mathrm{y} * *$ \\
\hline
\end{tabular}

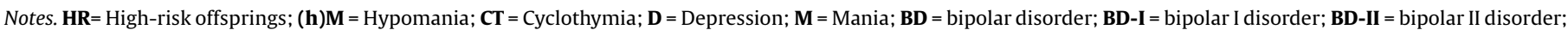

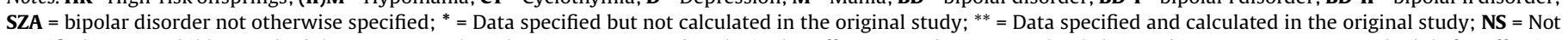

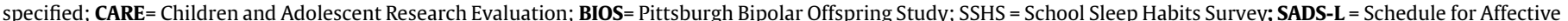

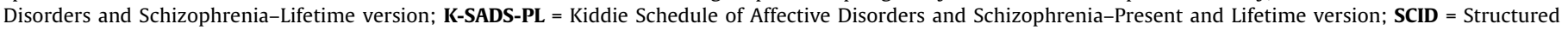
Clinical Interview for DSM-IV.

Table 2

Prospective studies with patients with sleep disorders/symptoms who develop Bipolar Disorder.

\begin{tabular}{|c|c|c|c|c|c|c|c|c|c|}
\hline \multirow[b]{2}{*}{ Study } & \multicolumn{2}{|l|}{ Design details } & \multicolumn{2}{|l|}{ Assessment } & \multirow[b]{2}{*}{$\begin{array}{l}\text { Follow-up } \\
\text { (years) }\end{array}$} & \multicolumn{2}{|c|}{ Age (years) } & \multicolumn{2}{|l|}{ Sleep prodromes } \\
\hline & Cohort & Sample & $\begin{array}{l}\text { Follow-up diagnosis / } \\
\text { scales }\end{array}$ & Sleep & & Baseline & $\begin{array}{l}1^{\text {st }} \\
\text { episode }\end{array}$ & Type / results & Preceding BD \\
\hline $\begin{array}{r}\text { Chung } \\
2015\end{array}$ & NHI /Taiwan & 30670 & BD / ICD-10 & ICD-9-CM & 6 & $>18$ & NS & Insomnia / HR= 14.69 & NS \\
\hline $\begin{array}{r}\text { Ritter } \\
2015\end{array}$ & $\begin{array}{l}\text { EDSP / } \\
\text { Germany }\end{array}$ & 1943 & $\begin{array}{l}\text { BD / DSM.IV, M-CIDI/DIA- } \\
\mathrm{X}\end{array}$ & $\begin{array}{l}\text { SCL-90 } \\
\text { Items }\end{array}$ & 10 & $18.3 \pm 3.6$ & $17.2 \pm 4.8$ & $\begin{array}{l}\text {-Trouble falling asleep / OR = } 1.51 \\
\text {-Early morning awakening / } \\
\mathrm{OR}=1.38\end{array}$ & $1.9 \mathrm{y}^{* *}$ \\
\hline
\end{tabular}

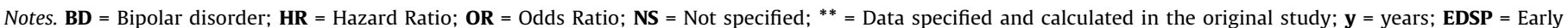

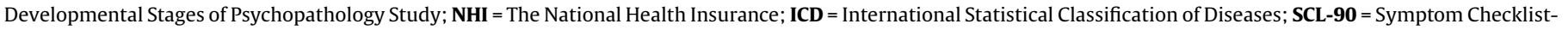
90-Revised; DSM-IV = Diagnostic and Statistical Manual of Mental Disorders-IV.

\subsubsection{Prospective studies on sleep disorders patients}

A total of 2 studies were included in this part of the review (Table 2).

The risk of psychiatric disorders over a 6 years follow-up period was evaluated in a large cohort study including adult subjects [34]. Patients with insomnia treated with hypnotic drugs had a higher risk of developing psychiatric disorders, especially BD, compared to those with insomnia not on hypnotics and people without insomnia too (HR: 7.60; 95\% CI: 5.31-10.89 and HR: 14.69; 95\% CI: 11.11-19.43).

The first study to assess longitudinally a possible association between disturbed sleep in healthy individuals and the subsequent onset of BD was conducted on a large cohort [35]. Among the 1943 patients included, 41 (1.8\%) developed BD at follow-up (mean age at onset $17.2 \pm 4.8$ years). Disturbed sleep in participants without a major mental disorder at T0 conferred an increased risk for BD onset (OR: 1.75; 95\% CI: $1.25-$ $2.45 ; \mathrm{p}=0.001$ ), even when adjusted for age, sex, parental mood disorder and lifetime cannabis or alcohol dependence. Even among participants without a positive family history of mood disorders $(N=1430)$ insomnia was significantly associated with $\mathrm{BD}(\mathrm{OR}=1.80 ; \mathrm{p}=0.003)$. When evaluating each sleep symptom, "trouble falling asleep" $(\mathrm{OR}=1.51 ; \mathrm{p}=0.006)$ and "early morning awakening" ( $O R=1.38 ; \mathrm{p}=0.048)$ showed significant odds of predicting BD. Survival analyses indicated that an increased risk over the 10 years follow-up with and a mean duration between T0 assessment and conversion to BD of 1.9 years.

\subsubsection{Retrospective studies on $B D$ patients}

A total of 11 studies were included in this part of the review (Table 3). 
Retrospective studies considering a history of sleep disorders/symptoms in BD patients.

\begin{tabular}{|c|c|c|c|c|c|c|c|c|c|c|c|c|}
\hline \multirow[b]{3}{*}{ Study } & \multicolumn{6}{|l|}{ Study Design } & \multicolumn{6}{|l|}{ Results } \\
\hline & \multicolumn{3}{|l|}{ Design details } & \multirow[b]{2}{*}{ Type } & \multicolumn{2}{|c|}{ Assessment } & \multicolumn{3}{|l|}{$\begin{array}{l}\text { Age } \\
\text { (years) }\end{array}$} & \multirow[b]{2}{*}{$\begin{array}{l}\text { Type of } 1^{\text {st }} \\
\text { episode }\end{array}$} & \multicolumn{2}{|l|}{ Sleep Prodromes } \\
\hline & Cohort & Sample & Study arms & & Diagnosis & Sleep & $\begin{array}{l}\text { Sleep } \\
\text { problems }\end{array}$ & $1^{\text {st }}$ episode & $\begin{array}{l}\text { BD } \\
\text { diagnosis }\end{array}$ & & Type/ frequency & Preceding BD \\
\hline $\begin{array}{l}\text { Egeland } \\
2000\end{array}$ & CARE /Amish & 58 & - BD-I & $\begin{array}{l}\text { Cross- } \\
\text { sectional }\end{array}$ & $\begin{array}{l}\text { RDC } \\
\text { (DSM-III) }\end{array}$ & $\begin{array}{l}\text { Medical records and } \\
\text { psychiatric } \\
\text { interviews }\end{array}$ & $13-15$ & $\begin{array}{l}\text { After } \\
\text { puberty }\end{array}$ & $\begin{array}{l}\text { After } \\
\text { puberty }\end{array}$ & NS & $\downarrow$ sleep / $24 \%$ & $9-12 y^{* *}$ \\
\hline $\begin{array}{r}\text { Faedda } \\
2004\end{array}$ & Private clinic / USA & $\begin{array}{l}82, \\
\text { pediatric }\end{array}$ & $-\mathrm{BD}$ & $\begin{array}{l}\text { Cross- } \\
\text { sectional }\end{array}$ & $\begin{array}{l}\text { Modified } \\
\text { DSM-IV } \\
\text { criteria }\end{array}$ & $\begin{array}{l}\text { K-SADS, parents, } \\
\text { medical records }\end{array}$ & $\begin{array}{l}<13 \text { in } \\
95 \%\end{array}$ & NS & $9.6 \pm 3.6$ & $\begin{array}{l}52.4 \% \mathrm{M}, 17.1 \% \\
\mathrm{D}, 30.5 \% \\
\text { mixed. }\end{array}$ & $\begin{array}{l}\text {-Insomnia and parasomnias / } \\
45.1 \%\end{array}$ & $\sim 7 y^{* *}$ \\
\hline $\begin{array}{r}\text { Correll } \\
2007\end{array}$ & $\begin{array}{l}\text { Child psychiatric center / } \\
\text { USA }\end{array}$ & 52 & $\begin{array}{l}\text { - psychotic BD (34) } \\
\text { - non psychotic BD } \\
\text { (18) }\end{array}$ & $\begin{array}{l}\text { Cross- } \\
\text { sectional }\end{array}$ & DSM-IV & BPSS-R & NS & $13.4 \pm 3.3$ & $13.4 \pm 3.3$ & $\begin{array}{l}36,5 \% \mathrm{M} \\
635 \% \mathrm{D}\end{array}$ & $\begin{array}{l}-\downarrow \text { need for sleep } \\
\text {-Insomnia }\end{array}$ & $\begin{array}{l}1.7 \pm 1.8 \text { y to } \\
1^{\text {st }} \text { psychotic } \\
\text { M ep. }{ }^{* *} \\
1.9 \pm 1.5 \text { y to } \\
1^{\text {st }} \\
\text { nonpsychotic } \\
\text { M ep. }{ }^{* *} \\
2.3 \pm 2.1 \text { y to } \\
1^{\text {st }} \text { D ep. }^{* *}\end{array}$ \\
\hline $\begin{array}{l}\text { Rucklidge } \\
2008\end{array}$ & $\begin{array}{l}\text { Clinical sample / New } \\
\text { Zealand }\end{array}$ & 82 & $\begin{array}{l}\text { - BD (25) } \\
\text { - ADHD (29) } \\
\text { - controls (28) }\end{array}$ & $\begin{array}{l}\text { Case- } \\
\text { control }\end{array}$ & $\begin{array}{l}\text { DSM-IV- } \\
\text { TR }\end{array}$ & Symptom Check List & $12-16$ & NS & $15-16$ & NS & $\begin{array}{l}\text {-Difficulty getting asleep / 60\% } \\
\text { - } \text { need for sleep / 32\% } \\
\text {-Frequently awakens at night / } \\
44 \%\end{array}$ & NS \\
\hline $\begin{array}{l}\text { Luckenbaugh } \\
2009\end{array}$ & $\begin{array}{l}\text { Child and adolescent } \\
\text { center / USA }\end{array}$ & 75 & $\begin{array}{l}\text { - BD }(27) \\
\text { - ADHD (22) } \\
\text { - controls }(26)\end{array}$ & $\begin{array}{l}\text { Case- } \\
\text { control }\end{array}$ & DSM-IV & Parent interview & NS & $7.2 \pm 4.1$ & $7.2 \pm 4.1$ & NS & $\downarrow$ sleep / 44\% & NS \\
\hline $\begin{array}{r}\text { Conus } \\
2010\end{array}$ & First Episode /Australia & 22 & $\begin{array}{l}-\operatorname{BD}(15) \\
-\operatorname{SZA}(7)\end{array}$ & $\begin{array}{l}\text { Cross- } \\
\text { sectional }\end{array}$ & DSM-IV & IMPQ & NS & $23 \pm 2.9$ & $23 \pm 2.9$ & $\begin{array}{l}100 \% \\
\text { psychotic M }\end{array}$ & $\begin{array}{l}\text {-Disrupted sleep / 83.3\% } \\
\text { - } \text { sleep or need for sleep / 61.1\% }\end{array}$ & $\begin{array}{l}20.9 \pm 16.4 \mathrm{w} \\
\text { to } 1^{\text {st }} \mathrm{M} \text { ep. }{ }^{* *}\end{array}$ \\
\hline $\begin{array}{l}\text { Skjelstad } \\
2011\end{array}$ & Outpatients / Norway & 15 & - BD-II & $\begin{array}{l}\text { Cross- } \\
\text { sectional }\end{array}$ & DSM-IV & Clinical Interview & $4.2 \pm 3.3$ & $15.9 \pm 3.6$ & $15.9 \pm 3.6$ & NS & $\begin{array}{l}\text {-Insomnia / 33.3\% } \\
\text {-Nightmares with violent } \\
\text { content / } 33.3 \% \\
\text {-Sleep walking / 33.3\% } \\
\text { - } \downarrow \text { need of sleep / 33.3\% }\end{array}$ & $\sim 11 \mathrm{y}^{*}$ \\
\hline $\begin{array}{l}\text { Zeschel } \\
2013\end{array}$ & Inpatients / Germany & 42 & $\begin{array}{l}\text { - BD-I (27) } \\
\text { - BD-II (15) }\end{array}$ & $\begin{array}{l}\text { Cross- } \\
\text { sectional }\end{array}$ & ICD-10 & BPSS-R & NS & $30.5 \pm 9.5$ & $30.5 \pm 9.5$ & $\begin{array}{l}21 \% \mathrm{M}, 786 \% \\
\mathrm{D}\end{array}$ & $\begin{array}{l}\text { - } \text { sleep requirement } \\
\text { - Insomnia } \\
\text { - Hypersomnia }\end{array}$ & $\begin{array}{l}1.5 \pm 1.9 \mathrm{~m} \text { to } \\
1^{\mathrm{st}} \mathrm{M} \text { ep. }{ }^{* *} \\
4.3 \pm 6.6 \mathrm{~m} \text { to } \\
1^{\text {st }} \mathrm{D} \mathrm{ep.}{ }^{* *}\end{array}$ \\
\hline $\begin{array}{l}\text { Salvatore } \\
2014\end{array}$ & $\begin{array}{l}\text { The McLean-Harvard } \\
\text { International First- } \\
\text { Episode Psychosis } \\
\text { Project / USA }\end{array}$ & 263 & - psychotic BD-I & $\begin{array}{l}\text { Cross- } \\
\text { sectional }\end{array}$ & $\begin{array}{l}\text { DSM-IV- } \\
\text { TR }\end{array}$ & $\begin{array}{l}\text { SCID-P, medical } \\
\text { records, interviews } \\
\text { with family and } \\
\text { clinicians }\end{array}$ & $18.0 \pm 0.0$ & $\begin{array}{l}-30.8 \pm 12.8 \\
1^{\text {st }} \mathrm{PE} \\
-22.3 \pm 10.7 \\
1^{\text {st }} \mathrm{NPE}\end{array}$ & $\begin{array}{l}\text { During } \\
\text { follow- } \\
\text { up }\end{array}$ & $\begin{array}{l}-23.5 \% 1^{\text {st }} \mathrm{PE} \\
-76.5 \% 1^{\text {st }} \\
\text { NPE }\end{array}$ & Sleep disturbances / 1.3\% & $16.5 \mathrm{y}$ to $1^{\text {st }} \mathrm{PE}$ \\
\hline $\begin{array}{l}\text { Kanady } \\
2015\end{array}$ & NIMH-funded trial / USA & 51 & - BD-I & $\begin{array}{l}\text { Cross- } \\
\text { sectional }\end{array}$ & $\begin{array}{l}\text { DSM-IV- } \\
\text { TR }\end{array}$ & NIMH interview & $18.3 \pm 9.7$ & $21.2 \pm 9.0$ & $21.2 \pm 9.0$ & NS & $\begin{array}{l}\text {-Insomnia } \\
\text {-Hypersomnia } \\
\text { - - sleep need } \\
\text {-Delayed sleep phase } \\
\text {-Irregular sleep patterns }\end{array}$ & $\sim 3 y^{*}$ \\
\hline $\begin{array}{l}\text { Noto } \\
2015\end{array}$ & Outpatients / Brazil & 43 & $\begin{array}{l}- \text { BD-I (32) } \\
\text { - BD-II (11) }\end{array}$ & $\begin{array}{l}\text { Cross- } \\
\text { sectional }\end{array}$ & DSM-IV & BPSS-R & NS & $25.3 \pm 8.1$ & $25.3 \pm 8.1$ & $\begin{array}{l}51.1 \% \text { D, } 32.5 \% \\
\text { M, 4.6\% (h)M, } \\
11.6 \% \text { mixed }\end{array}$ & $\begin{array}{l}\text {-Insomnia } \\
\text { - \need for sleep } \\
\text {-Inversion of the sleep/ } \\
\text { wakefulness pattern } \\
\text {-Hypersomnia }\end{array}$ & $1-16.7 m^{* *}$ \\
\hline
\end{tabular}

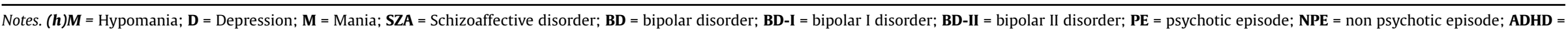

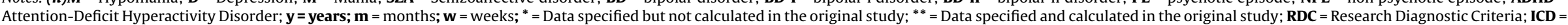

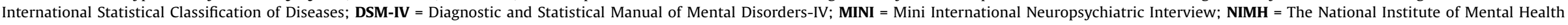

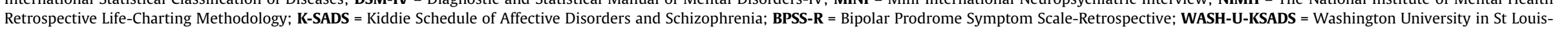
Schedule for Affective Disorders and Schizophrenia; CARE= Children and Adolescent Research Evaluation; IMPQ = Initial Mania Prodrome Questionnaire. 
In the aforementioned Amish study, psychopathological features of parent patients prior to BDdiagnosis were asked to recall prodromal symptopms [36]. Sleep problems appeared as the fourth most common symptoms reported between age 13 and 15 (23\%), detectable during childhood at least 9-12 years prior to BD onset.

Another study evaluated 82 pediatric BD patients [37]. About 7 years of delay were found until BD diagnosis/treatment (symptomatic onset occurred before age 3 in $74 \%$ of cases, and before age 13 in 95\%).

Potential temporal and symptomatic differences between the prodrome to psychotic vs. nonpsychotic manic onsets were assessed in a study including a sample of $52 \mathrm{BD}$ children and adolescents [38]. Non significant differences in prevalence in psychotic mania vs. non psychotic mania for: "decreased need for sleep" (respectively $41.2 \%$ vs. $33.3 \%, \mathrm{p}=0.58$ ) and "insomnia" ( $41.2 \%$ vs. $27.8 \%, p=0.34)$. Both groups presented a prevalent "insidious" pattern of onset of prodromal symptoms $(1.7 \pm 1.8$ years before psychotic mania, $1.9 \pm 1.5$ years before nonpsychotic mania, $2.3 \pm 2.1$ before depressiion).

A comparison between Attention Deficit Hyperactivity Disorder (ADHD, $n=29), B D(n=25)$ and healthy controls $(n=28)$ aimed at investigating possible prodromal symptoms to these conditions in adolescents [39]. Among BD patients, 60\% had difficulties getting to sleep and $32 \%$ presented a decreased need for sleep in adolescence compared respectively to $65.5 \%$ and $24.1 \%$ (ADHD group) and $28.6 \%$ and $3.6 \%$ (control group) with no statistical significance. "Frequent awakenings at night" was present in $44 \%$ of BD patients and $7.1 \%$ of the control group $(p<0.01)$.

The course of individual symptoms over the first 10 years of life in juvenile-onset BD and ADHD, alone or comorbid, was compared with healthy controls [40]. "Decreased sleep" was present in $44 \%$ of the $\mathrm{BD}, 9 \%$ of the ADHD, $8 \%$ of controls, and significantly higher in BD vs. ADHD $(p=0.0005)$ and VD vs. controls $(p=n r)$.

Another study examined symptoms and prodromes 1 year before a psychotic manic onset [41]. Clinical symptoms were common in $11 / 22$ (50\%) participants. Among them, "disrupted sleep" was present in $83.3 \%$ of the sample, "reduced sleep or need for sleep" in $61.1 \%$. The mean duration of sleep symptoms was $20.9 \pm 16.4$ weeks.

In a Norwegian study, prodromal symptoms and behaviours of 15 BD type II patients were explored [42]. Prodromal symptoms were divided into 3 groups according to whether life events at the time of the symptom elicited an appropriate or exaggerated reaction: group $\mathrm{A}$, with symptoms linked to normal responses to environment stimuli, group $B$, with symptoms characterized as "exaggerated" in relation to environment stimuli, group $\mathrm{C}$, with symptoms characterized as inexplicable or unrelated to the context or to environment stimuli. Symptoms of B and C criteria. Overall, 5/15 patients experimented sleep disturbances. No patients with sleep disturbances met the B or C criteria. Sleep symptoms started about 11 years before BD.

The course of pre-(hypo)manic and pre-depressed prodromal symptoms was assessed in a study on 44 BD type I or II patients [43]. "Reduced sleep requirement" was present $1.3 \pm 1.9$ months prior to illness onset in $71.4 \%$ of the pre-(hypo)manic patients and none of the pre-depressed. "Insomnia" was present $1.4 \pm 2.0$ months before in $54.8 \%$ of the pre-(hypo)manic patients and in $66.7 \%$ of the pre-depressed ( $3.9 \pm 6.9$ months). "Hypersomnia" was present $1.0 \pm 1.1$ months prior in $7.1 \%$ of the pre-(hypo)manic patients and $6.0 \pm 9.7$ months before in $33.3 \%$ of the pre-depressed.

One of the studies proceeding from the McLean-Harvard First Episode Project investigated possible differences in pre-onset symptoms between purely psychotic- $(23.5 \%)$, and manic psychotic- (18.1\%) and depressive psychotic (58.4\%) onset (76.5\%) patients in $263 \mathrm{BD}-\mathrm{I}$ patients with at least one lifetime psychotic episode,
[44]. "Sleep disturbances" were present in $1.3 \%$ of the psychotic manic-onset group and absent in the others $(p=n s)$ Age at first sleep problem was $18.0 \pm 0.0$ years, about 16.5 years before the onset of the first psychotic episode.

A recent study aimed to retrospectively examine the prevalence, coexistence and persistence of sleep disturbances across the course of BD [45]. Sleep disturbances (i.e. insomnia, hypersomnia, reduced need for sleep, delayed sleep phase and irregular sleep patterns) preceded the onset of illness of about 3 years (age at onset of sleep disturbance: $18.3 \pm 9.7$ ).

The prodromes of BD (hypo)manic vs. depressive onset were studied on a sample of 43 stable BD type I (74.4\%) and II (25.6\%) outpatients [46]. Prior to (hypo)manic onset, "insomnia" was present in $48.8 \%$, "decreased need of sleep" in $25.6 \%$, the "inversion of the sleep/wakefulness pattern" in 14\% and hypersomnia in $7 \%$. Prior to depressive onset, "insomnia" and "hypersomnia" were both present in 14\% of BD subjects, "decreased need for sleep" and "inversion of sleep/wakefulness pattern" both in 4.6\%. Sleep problems were more frequent prior to (hypo)manic onset than to depressive onset, independently of BD subtype.

The quality assessment of the studies included in this systematic review outlines a wide heterogeneity in studies design, populations and outcomes (See Suppl. Table 1). Among the cohort studies, 2 groups showed true representativeness. All cohort studies showed adequate case definition groups of interest appropriate evaluation of outcomes and good follow-up. The quality evaluation of case-control studies returned lower quality in representativeness, sample size and comparability, and - by definition- lower quality in outcome recollection, limited by selfreport.

\section{Discussion}

This systematic review aimed at understanding the clinical relationship between sleep problems and the onset of $\mathrm{BD}$, updating the work by Ritter and cols. (2011).

In the last years several new studies focused specifically on this topic, 4 reporting results on prospective follow-ups [32-35], whilst 11 presenting retrospective design. The onset of sleep problems in people who subsequently developed BD may long anticipate a fullblown $\mathrm{BD}$, occurring during adolescence or pre-adolescence $[1,20,31]$. These alterations may be pointed out with subjective and objective (i.e. actigraphic) measures also in populations at high-risk for BD, but never diagnosed [47]. Sleep and chronotype alteration patterns are similar to those found in full-blown BD patients when compared to healthy controls [48], so that their accuracy in predicting a conversion to full-blown BD has to be ascertained.

Globally, sleep problems seem more frequent in the offspring of patients with BD (high-risk offspring) compared to children of healthy controls [49], with a surprising 30 -fold increased risk to develop sleep disorders compared to not-at-risk offspring [31]. Despite the clear association, no threshold on sleep alterations patterns has been outlined. Also, high-risk offspring present weak and more unstable rest-activity cycles, as indicated with actigraphy by lower relative amplitude and higher variability in sleep efficiency in comparison with controls [9]. Unfortunately, clinical applicability of these findings is doubtful.

When taking into consideration the lapse between sleep disturbances and BD onset, prospective studies almost invariantly agree on the development of generic sleep problems more than 1 year before BD (1.9-6 years [50,32,33,35]. Interestingly, the retrospective studies reviewed present a longer latency, more than 1 year before BD onset (adolescence or pre-adolescence) $[37,38,42,44]$ compared to few months in young adulthood onset $[41,43,46]$. An overestimation of sleep problems is possible. Also, 
generalizability from child-adolescent BD to adult BD populations may be hampered by the lack lack of consensus over the very consistence of BD diagnosis in childhood/adolescence [51,52].

\subsection{Sleep prodromes and polarity of onset}

\subsubsection{Decreased need for sleep}

A common finding throughout the present review is that decreased sleep may be present time before BD onset in a variable percentage of patients ranging from $24 \%$ to $44 \%$ in retrospective studies $[36,39,40]$, and up to $50 \%$ in a prospective study [30]. This seems an aspecific finding, as, for instance, a generic decrease in sleep also is reported for individuals at risk for psychosis with no significant differences compared to individuals at risk for BD [7]. Undoubtley a close monitoring of individuals at risk for developing both conditions is due anyway, and the treatment of common psychopathologica prodromes could be tried as suggested by recent transdiagnostic staging models [4]. Predictably, manic and hypomanic BD onset episodes are mostly associated with a decreased need for sleep, appearing in $25.6 \%$ up to $71.4 \%$ of premanic patients $[43,46]$ and it may precede BD manic onset by about 1-8 months. According to the results of this review, prolonged decreased need for sleep represents a good prodrome to a manic onset.

\subsubsection{Insomnia}

Insomnia seems an important prodrome of $\mathrm{BD}$ in 2 prospective studies, with an HR of 14.69 in one study [34], and with an OR of 1.51 (for initial insomnia) and 1.38 (for late insomnia) in the other [35]. Also retrospective studies seem consistent with its predictive value. Insomnia seems the most frequent symptom prior to depressive onset (14.0\%-66.7\%), and appearing 7.3 to 3.9 months before the episode $[35,43,46]$. Insomnia also precede manic onset $(48.8 \%-54.8 \%)$ from 1.9 months up to $1.9 \pm 1.5$ years $[38,43,46]$. Again, insomnia is very unspecific, also commonprior to unipolar depression [20,53,54]. Despite its aspecific nature, insomnia allows identification of populations at high risk for serious mental conditions such as BD, depression or schizophrenia.

\subsubsection{Hypersomnia}

Although less frequent, hypersomnia seems more specific of a depressive $\mathrm{BD}$ onset. It precedes the onset of the disease of 67 months $[43,46]$, its frequency ranging from $14 \%$ up to $33.3 \%$, compared with pre-manic patients (about 7\%). Hypersomnia was associated with the onset of a bipolar depression episode [55] and may also differentiate bipolar depression (in BD type II patients) from unipolar depression, with a positive predictive value of around 70\% [56]. Interestingly, hypersomnia is also a clinical symtptom present in the "atypical features" diagnostic specifier. This underlines its possible direct and indirect relation with a bipolar diathesis in major depression that is being investigated [57]. Despite a careful and critical assessment of hypersomnia is due [58], our results suggest that hypersomnia is a potential prodrome of bipolar depressive onset.

\subsubsection{Circadian rhythm alterations}

In this review scant data concerning alterations in circadian rhythms were returned. A lower likelihood of conversion for the "extreme evening type" pattern of circadian rhythm (i.e. defined as an eveningness circadian preference $<10$ th percentile of the entire sample) in youth at genetic risk for BD was reported in one study [59]. This contrasts with previous evidence, as BD in adolescent and adult BD patients seems significantly associated with significantly higher prevalence of "evening types" than healthy controls $[10,60,61]$. However, it circadian preference tends to differ between childhood and adolescence and a substantial shift to an evening circadian preference is observed at around 12-13 years of age [62,63]. Considering that the mean age in the study by Levenson and cols. was $11 \pm 3.6$ years, it is possible that a switch to an evening chronotype was missed. Also, the association of evening chronotype and BD could be independent or partially mediated by chronic sleep deprivation due to social needs (e.g. early awakening) triggering a mood episode [64]. One included study reports a generic "inversion of the sleep/wakefulness pattern". This is not frequent in either pre-depressive and premanic patients, but when present it might anticipate full illnessonset more than one year. [46]. Newest resultsfrom the BIOS cohort [32] show that weekend-to-weekday sleep variability were not substantially associated with elevated risk of developing BD among at-risk youth.

To a varying degree, results in this systematic review point to somewhat aspecific sleep and biological rhythm disturbances. Despite this, their appearance in at-risk populations and their high prevalence anticipating acute episodes $[65,66]$, calls for specific and timely interventions. This is especially true when considering that sleep alterations are potentially amenable to pharmacological and psychological treatment [67]. To avoid overmedication of these individuals, psychological and psychoeducational interventions should be especially considered to achieve improved illness outcomes [68-70].

\section{Limitations}

The present systematic review was conducted considering only studies in English language. Although this potentially limits the generalizability of results in terms of geographic and ethnical differences, most international peer-review journals publish studies written in English language

We also chose to include data on diagnosed BD patients. This is likely to have cut out most of the evidence on sleep biomarkers and translational research performed on high-risk individuals. On the other hand, the excluded evidence could point to populations at risk and be not associated with a full-blown BD condition, and this could be especially true for child/adolescent BD populations due to diagnostic consistency in such populations.

Also, studies focused on specific subpopulations such as BD type II or rapid cycling BD are lacking

The predictive value of sleep disturbances seems somewhat hampered by the scarcity of prospective studies on the topic and the heterogeneity of assessment measures used. Retrospective studies present data with possible recollection bias

\section{Conclusions}

Sleep prodromes seem good potential indicators for the early detection in those at highest risk of developing bipolar disorder. Their increased prevalence may be detected more than 1 year prior to the onset of the first affective episode. Despite sleep prodromes overall lack specificity for BD, especially in a pure clinical setting, hypersomnia might have a possible role in discriminating bipolar versus unipolar depression, and reduced need for sleep be a useful indicator for a manic onset. Early recognition and early specific intervention on sleep disturbance allow for a most effective management, but might also allow postpone illness onsetduring crucial periods of life such as adolescence and young adulthood.

Sleep and chronotype research still need more objective quantitative and qualitative approaches such as actigraphy and polysomnography evaluations or neuroendocrine assessment.

\section{Acknowledgements}

The authors thank the support of the Spanish Ministry of Science, Innovation and Universities integrated into the Plan 
Nacional de I+D+I and co-financed by the ISCIII-Subdirección General de Evaluación and the Fondo Europeo de Desarrollo Regional (FEDER); the CIBERSAM (Centro de Investigación Biomédica en Red de Salud Mental); the Secretaria d'Universitats i Recerca del Departament d'Economia i Coneixement (2017_SGR_1365) and the CERCA Programme / Generalitat de Catalunya.

\section{Appendix A. Supplementary data}

Supplementary material related to this article can be found, in the online version, at doi:10.1016/j.eurpsy.2019.02.003.

\section{References}

[1] Benarous X, Consoli A, Milhiet V, Cohen D. Early interventions for youths at high risk for bipolar disorder: a developmental approach. Eur Child Adolesc Psychiatry 2016;25(Mar (3)):217-33, doi:http://dx.doi.org/10.1007/s00787015-0773-6.

[2] Faedda GL, Marangoni C, Serra G, Salvatore P, Sani G, Vázquez GH, et al. Precursors of bipolar disorders: a systematic literature review of prospective studies. J Clin Psychiatry 2015;76(May (5)):614-24, doi:http://dx.doi.org/ 10.4088/JCP.13r08900

[3] Vieta E, Berk M, Schulze TG, Carvalho AF, Suppes T, Calabrese JR, et al. Bipolar disorders. Nat Rev Dis Prim 2018;(Mar 4):18008, doi:http://dx.doi.org/ 10.1038/nrdp.2018.8.

[4] Scott J, Leboyer M, Hickie I, Berk M, Kapczinski F, Frank E, et al. Clinical staging in psychiatry: a cross-cutting model of diagnosis with heuristic and practical value. Br J Psychiatry 2013;202(Apr (4)):243-5, doi:http://dx.doi.org/10.1192/ bjp.bp. 112.110858.

[5] Vieta E, Salagre E, Grande I, Carvalho AF, Fernandes BS, Berk M, et al. Early intervention in bipolar disorder. Am J Psychiatry 2018;175(May (5)):411-26, doi:http://dx.doi.org/10.1176/appi.ajp.2017.17090972.

[6] Ritter PS, Marx C, Lewtschenko N, Pfeiffer S, Leopold K, Bauer M, et al. The characteristics of sleep in patients with manifest bipolar disorder, subjects at high risk of developing the disease and healthy controls. J Neural Transm 2012;119(Oct (10)):1173-84, doi:http://dx.doi.org/10.1007/ s00702-012-0883-y.

[7] Zanini MA, Castro J, Cunha GR, Asevedo E, Pan PM, Bittencourt L, et al. Abnormalities in sleep patterns in individuals at risk for psychosis and bipolar disorder. Schizophr Res 2015;169(Dec (1-3)):262-7, doi:http://dx.doi.org/ 10.1016/j.schres.2015.08.023.

[8] APA. Diagnostic and statistical manual of mental disorders. fifth edition 2013 (DSM-5). APA, editor. Arlington VA.

[9] Ng TH, Chung K-F, FY-Y Ho, Yeung W-F, Yung K-P, Lam T-H. Sleep-wake disturbance in interepisode bipolar disorder and high-risk individuals: a systematic review and meta-analysis. Sleep Med Rev 2015;20(Apr):46-58, doi:http://dx.doi.org/10.1016/j.smrv.2014.06.006.

[10] Milhiet V, Boudebesse C, Bellivier F, Drouot X, Henry C, Leboyer M, et al. Circadian abnormalities as markers of susceptibility in bipolar disorders. Front Biosci (Schol Ed) 2014;6:120-37.

[11] Salvadore G, Quiroz JA, Machado-Vieira R, Henter ID, Manji HK, Zarate CA. The neurobiology of the switch process in bipolar disorder: a review. J Clin Psychiatry 2010;71(Nov (11)):1488-501, doi:http://dx.doi.org/10.4088/ JCP.09r05259gre.

[12] Bauer M, Juckel G, Correll CU, Leopold K, Pfennig A. Diagnosis and treatment in the early illness phase of bipolar disorders. Eur Arch Psychiatry Clin Neurosci 2008;258(Suppl):50-4.

[13] Young JW, Dulcis D. Investigating the mechanism(s) underlying switching between states in bipolar disorder. Eur J Pharmacol 2015;(Jul 759):151-62, doi: http://dx.doi.org/10.1016/j.ejphar.2015.03.019.

[14] Pinho M, Sehmbi M, Cudney LE, Kauer-Sant'anna M, Magalhães PV, Reinares M, et al. The association between biological rhythms, depression, and functioning in bipolar disorder: a large multi-center study. Acta Psychiatr Scand 2015 (May), doi:http://dx.doi.org/10.1111/acps.12442.

[15] Pagani L, St Clair PA, Teshiba TM, Service SK, Fears SC, Araya C, et al. Genetic contributions to circadian activity rhythm and sleep pattern phenotypes in pedigrees segregating for severe bipolar disorder. Proc Natl Acad Sci U S A 2016:113(Feb (6)):E754-61, doi:http://dx doi.org/10.1073/pnas.1513525113.

[16] Geoffroy PA, Scott J. Prodrome or risk syndrome: what's in a name? Int J Bipolar Disord 2017;5(Dec (1)):7, doi:http://dx.doi.org/10.1186/s40345-0170077-5.

[17] Kapczinski F, Magalhães PVS, Balanzá-Martinez V, Dias VV, Frangou S, Gama CS, et al. Staging systems in bipolar disorder: an international society for bipolar disorders task force report. Acta Psychiatr Scand 2014;130(Nov (5)):354-63, doi:http://dx.doi.org/10.1111/acps.12305.

[18] Alda M, Kapczinski F. Staging model raises fundamental questions about the nature of bipolar disorder. J Psychiatry Neurosci 2016;41(Sep (5)):291-3, doi: http://dx.doi.org/10.1503/jpn.160151.

[19] Carvalho AF, Köhler CA, Fernandes BS, Quevedo J, Miskowiak KW, Brunoni AR, et al. Bias in emerging biomarkers for bipolar disorder. Psychol Med 2016;46 (Aug (11)):2287-97, doi:http://dx.doi.org/10.1017/S0033291716000957.
[20] Ritter PS, Marx C, Bauer M, Leopold K, Lepold K, Pfennig A. The role of disturbed sleep in the early recognition of bipolar disorder: a systematic review. Bipolar Disord 2011;13(May (3)):227-37, doi:http://dx.doi.org/10.1111/j.13995618.2011.00917x.

[21] Moher D, Liberati A, Tetzlaff J, Altman DG, PRISMA Group. Preferred reporting items for systematic reviews and meta-analyses: the PRISMA statement. BM] 2009;339:b2535.

[22] American Psychiatric Association. . Diagnostic and statistical manual of mental disorders: DSM-III American Psychiatric Association, editor. Washington, DC. 1987.

[23] American Psychiatric Association. Diagnostic and statistical manual of mental disorders: DSM-IV American Psychiatric Association, editor. Washington, DC 1994.

[24] American Psychiatric Association. Diagnostic and statistical manual of mental disorders: DSM-IV-TR American Psychiatric Association, editor. Washington, DC. 2000.

[25] World Health Organization. International statistical classification of diseases and health related problems. eighth edition 1967 World Health Organization., editor.

[26] World Health Organization. International statistical classification of diseases and health related problems. ninth edition 1977.

[27] World Health Organization. International statistical classification of diseases and health related problems. tenth edition 1997.

[28] Zeng X, Zhang Y, Kwong JSW, Zhang C, Li S, Sun F, et al. The methodological quality assessment tools for preclinical and clinical studies, systematic review and meta-analysis, and clinical practice guideline: a systematic review. J Evid Based Med 2015;8(Feb (1)):2-10, doi:http://dx.doi.org/10.1111/ jebm.12141.

[29] Herzog R, Álvarez-Pasquin MJ, Díaz C, Del Barrio JL, Estrada JM, Gil Á. Are healthcare workers' intentions to vaccinate related to their knowledge, beliefs and attitudes? a systematic review. BMC Public Health 2013;13(Dec (1)):154 doi:http://dx.doi.org/10.1186/1471-2458-13-154.

[30] Egeland JA, Endicott J, Hostetter AM, Allen CR, Pauls DL, Shaw JA. A 16-year prospective study of prodromal features prior to BPI onset in well Amish children. J Affect Disord 2012;142(Dec (1-3)):186-92, doi:http://dx.doi.org/ 10.1016/j.jad.2012.04.023.

[31] Duffy A, Horrocks J, Doucette S, Keown-Stoneman C, McCloskey S, et al. The developmental trajectory of bipolar disorder. Br J Psychiatry 2014;204(Feb (2)):122-8, doi:http://dx.doi.org/10.1192/bjp.bp.113.126706.

[32] Levenson JC, Soehner A, Rooks B, Goldstein TR, Diler R, Merranko J, et al. Longitudinal sleep phenotypes among offspring of bipolar parents and community controls. J Affect Disord 2017;6(Mar 215):30-6, doi:http://dx.doi. $\operatorname{org} / 10.1016 / j . j a d .2017 .03 .011$.

[33] Mesman E, Nolen WA, Keijsers L, Hillegers MHJ. Baseline dimensional psychopathology and future mood disorder onset: findings from the Dutch Bipolar Offspring Study. Acta Psychiatr Scand 2017;136(Aug (2)):201-9, doi: http://dx.doi.org/10.1111/acps.12739.

[34] Chung K-H, Li C-Y, Kuo S-Y, Sithole T, Liu W-W, Chung M-H. Risk of psychiatric disorders in patients with chronic insomnia and sedative-hypnotic prescription: a nationwide population-based follow-up study. J Clin Sleep Med 2015;11(May (5)):543-51, doi:http://dx.doi.org/10.5664/jcsm.4700.

[35] Ritter PS, Höfler M, Wittchen H-U, Lieb R, Bauer M, Pfennig A, et al. Disturbed sleep as risk factor for the subsequent onset of bipolar disorder-Data from a 10-year prospective-longitudinal study among adolescents and young adults. J Psychiatr Res 2015;68(Sep):76-82, doi:http://dx.doi.org/10.1016/j. jpsychires.2015.06.005

[36] Egeland JA, Hostetter AM, Pauls DL, Sussex JN. Prodromal symptoms before onset of manic-depressive disorder suggested by first hospital admission histories. J Am Acad Child Adolesc Psychiatry 2000;39(Oct (10)):1245-52, doi http://dx.doi.org/10.1097/00004583-200010000-00011.

[37] Faedda GL, Baldessarini RJ, Glovinsky IP, Austin NB. Pediatric bipolar disorder: phenomenology and course of illness. Bipolar Disord 2004;6(Aug (4)):305-13, doi:http://dx.doi.org/10.1111/j.1399-5618.2004.00128.x.

[38] Correll CU, Penzner JB, Frederickson AM, Richter JJ, Auther AM, Smith CW, et al. Differentiation in the preonset phases of schizophrenia and mood disorders: evidence in support of a bipolar mania prodrome. Schizophr Bull 2007;33 (3):703-14.

[39] Rucklidge JJ. Retrospective parent report of psychiatric histories: do checklists reveal specific prodromal indicators for postpubertal-onset pediatric bipolar disorder? Bipolar Disord 2008;10(Feb (1)):56-66, doi:http://dx.doi.org/ 10.1111/j.1399-5618.2008.00533.x.

[40] Luckenbaugh DA, Findling RL, Leverich GS, Pizzarello SM, Post RM. Earliest symptoms discriminating juvenile-onset bipolar illness from ADHD. Bipolar Disord 2009;11(June (4)):441-51, doi:http://dx.doi.org/10.1111/j.13995618.2009.00684.x.

[41] Conus P, Ward J, Lucas N, Cotton S, Yung AR, Berk M, et al. Characterisation of the prodrome to a first episode of psychotic mania: results of a retrospective study. J Affect Disord 2010;124(3):341-5.

[42] Skjelstad DV, Holte A, Malt UF. Genuine clinical predictors of bipolar II disorder: an exploration of temporal and contextual characteristics. J Affect Disord 2011;135(December (1-3)):419-23, doi:http://dx.doi.org/10.1016/j. jad.2011.08.029.

[43] Zeschel E, Correll CU, Haussleiter IS, Krüger-Özgürdal S, Leopold K, Pfennig A, et al. The bipolar disorder prodrome revisited: Is there a symptomatic pattern? J Affect Disord 2013;151(November (2)):551-60, doi:http://dx.doi.org/ 10.1016/j.jad.2013.06.043. 
[44] Salvatore P, Baldessarini RJ, H-MK Khalsa, Vázquez G, Perez J, Faedda GL, et al. Antecedents of manic versus other first psychotic episodes in 263 bipolar I disorder patients. Acta Psychiatr Scand 2014;129(April (4)):275-85, doi: http://dx.doi.org/10.1111/acps.12170.

[45] Kanady JC, Soehnera AM, Harvey AG. A retrospective examination of sleep disturbance across the course of bipolar disorder. J Sleep Disord Ther 2015;4 (March (2)), doi:http://dx.doi.org/10.4172/2167-0277.1000193.

[46] Noto MN, Noto C, Caribé AC, Miranda-Scippa Â, Nunes SO, Chaves AC, et al. Clinical characteristics and influence of childhood trauma on the prodrome of bipolar disorder. Rev Bras Psiquiatr (São Paulo, Brazil 1999) 2015;37(4):280-8, doi:http://dx.doi.org/10.1590/1516-4446-2014-1641.

[47] Melo MCA, Garcia RF, Linhares Neto VB, Sá MB, de Araújo CFC, et al. Sleep and circadian alterations in people at risk for bipolar disorder: a systematic review. J Psychiatr Res 2016;13(Sep 83):211-9, doi:http://dx.doi.org/10.1016/j. jpsychires.2016.09.005.

[48] De Crescenzo F, Economou A, Sharpley AL, Gormez A, Quested DJ. Actigraphic features of bipolar disorder: a systematic review and meta-analysis. Sleep Med Rev 2016.

[49] Sebela A, Novak T, Kemlink D, Goetz M. Sleep characteristics in child and adolescent offspring of parents with bipolar disorder: a case control study. BMC Psychiatry 2017;17(Dec (1)):199, doi:http://dx.doi.org/10.1186/s12888017-1361-8.

[50] Duffy A, Alda M, Hajek T, Sherry SB, Grof P. Early stages in the development of bipolar disorder. J Affect Disord 2010;121(Feb (1-2)):127-35, doi:http://dx. doi.org/10.1016/j.jad.2009.05.022.

[51] Carlson GA, Meyer SE. Phenomenology and diagnosis of bipolar disorder in children, adolescents, and adults: complexities and developmental issues. Dev Psychopathol 2006;18(Dec (4)):939-69, doi:http://dx.doi.org/10.1017/ S0954579406060470.

[52] Douglas J, Scott J. A systematic review of gender-specific rates of unipolar and bipolar disorders in community studies of pre-pubertal children. Bipolar Disord 2014;16(Feb (1)):5-15, doi:http://dx.doi.org/10.1111/bdi.12155.

[53] Baglioni C, Battagliese G, Feige B, Spiegelhalder K, Nissen C, Voderholzer U, et al. Insomnia as a predictor of depression: a meta-analytic evaluation of longitudinal epidemiological studies. J Affect Disord 2011;135(Dec (1-3)):109, doi:http://dx.doi.org/10.1016/j.jad.2011.01.011.

[54] Correll CU, Penzner JB, Frederickson AM, Richter JJ, Auther AM, Smith CW, et al. Differentiation in the preonset phases of schizophrenia and mood disorders: evidence in support of a bipolar mania prodrome. Schizophr Bull 2007;33(May (3)):703-14, doi:http://dx.doi.org/10.1093/schbul/sbm028.

[55] Kaplan KA, Gruber J, Eidelman P, Talbot LS, Harvey AG. Hypersomnia in interepisode bipolar disorder: does it have prognostic significance? J Affect Disord 2011;132(Aug (3)):438-44, doi:http://dx.doi.org/10.1016/j.jad.2011.03.013.

[56] Hantouche EG, Akiskal HS. Bipolar II vs. Unipolar depression: psychopathologic differentiation by dimensional measures. J Affect Disord 2005;84(Feb (2-3)):127-32, doi:http://dx.doi.org/10.1016/j.jad.2004.01.017.

[57] Petri E, Bacci O, Barbuti M, Pacchiarotti I, Azorin J-M, Angst J, et al. Obesity in patients with major depression is related to bipolarity and mixed features: evidence from the BRIDGE-II-Mix study. Bipolar Disord 2017;19(Sep (6)):45864, doi:http://dx.doi.org/10.1111/bdi.12519.

[58] Dauvilliers Y, Lopez R, Ohayon M, Bayard S. Hypersomnia and depressive symptoms: methodological and clinical aspects. BMC Med 2013;11(Mar):78, doi:http://dx.doi.org/10.1186/1741-7015-11-78.

[59] Levenson JC, Axelson DA, Merranko J, Angulo M, Goldstein TR, Mullin BC, et al. Differences in sleep disturbances among offspring of parents with and without bipolar disorder: association with conversion to bipolar disorder. Bipolar Disord 2015;17(Dec (8)):836-48, doi:http://dx.doi.org/10.1111/bdi.12345.
[60] Wood J, Birmaher B, Axelson D, Ehmann M, Kalas C, Monk K, et al. Replicable differences in preferred circadian phase between bipolar disorder patients and control individuals. Psychiatry Res 2009;166(Apr (2-3)):201-9, doi:http://dx. doi.org/10.1016/j.psychres.2008.03.003.

[61] Kim KL, Weissman AB, Puzia ME, Cushman GK, Seymour KE, Wegbreit E, et al. Circadian phase preference in pediatric bipolar disorder. J Clin Med 2014;3 (1):255-66, doi:http://dx.doi.org/10.3390/jcm3010255.

[62] Gau S-F, Soong W-T. The transition of sleep-wake patterns in early adolescence. Sleep 2003;26(Jun (4)):449-54.

[63] Tonetti L, Adan A, Di Milia L, Randler C, Natale V. Measures of circadian preference in childhood and adolescence: a review. Eur Psychiatry 2015;30 (5):576-82, doi:http://dx.doi.org/10.1016/j.eurpsy.2015.01.006.

[64] Melo MCA, Abreu RLC, Linhares Neto VB, de Bruin PFC, de Bruin VMS. Chronotype and circadian rhythm in bipolar disorder: A systematic review. Sleep Med Rev 2016(July), doi:http://dx.doi.org/10.1016/j.smrv.2016.06.007.

[65] Jackson A, Cavanagh J, Scott J. A systematic review of manic and depressive prodromes. J Affect Disord 2003;74(May (3)):209-17.

[66] Rosa AR, Comes M, Torrent C, Solè B, Reinares M, Pachiarotti I, et al. Biological rhythm disturbance in remitted bipolar patients. Int J Bipolar Disord 2013;1:6, doi:http://dx.doi.org/10.1186/2194-7511-1-6.

[67] Joslyn C, Hawes DJ, Hunt C, Mitchell PB. Is age of onset associated with severity, prognosis, and clinical features in bipolar disorder? A meta-analytic review. Bipolar Disord 2016;18(Aug (5)):389-403, doi:http://dx.doi.org/10.1111/ bdi.12419.

[68] Oud M, Mayo-Wilson E, Braidwood R, Schulte P, Jones SH, Morriss R, et al. Psychological interventions for adults with bipolar disorder: systematic review and meta-analysis. Br J Psychiatry 2016;208(Mar (3)):213-22, doi: http://dx.doi.org/10.1192/bjp.bp.114.157123.

[69] Vieta E, Morilla I. Early group psychoeducation for bipolar disorder. Lancet Psychiatry 2016(Sep), doi:http://dx.doi.org/10.1016/S2215-0366(16)30303-0.

[70] Vieta E, Salagre E, Grande I, Carvalho AF, Fernandes BS, Berk M, et al. Early intervention in bipolar disorder. Am J Psychiatry 2018(Jan), doi:http://dx.doi. org/10.1176/appi.ajp.2017.17090972.

\section{Glossary}

$S A D S-L$ : Schedule for Affective Disorders and Schizophrenia-Lifetime version K-SADS: Affective Disorders module of the Schedule for Affective Disorders and Schizophrenia for School-Age Children

K-SADS-PL: Kiddie Schedule of Affective Disorders and Schizophrenia-Present and Lifetime version

WASH-U-KSADS: Washington University in St Louis Schedule for Affective Disorders and Schizophrenia

BPSS-R: Bipolar Prodrome Symptom Scale-Retrospective

$A D H D$ : attention-deficit hyperactivity disorder

$B D$ : Bipolar Disorder

BD-I/BD-II: Bipolar I Disorder/Bipolar II Disorder

SCID: Structured Clinical Interview for DSM-IV

DSM-IV: Diagnostic and Statistical Manual of Mental Disorders-IV

ICD-10: The International Classification of Diseases-10

AHR: Adjusted Hazard Ratio

OR: Odds Ratio 